\title{
Characterization of the invasive and inflammatory traits of oral Campylobacter rectus in a murine model of fetoplacental growth restriction and in trophoblast cultures
}

\author{
R.M. Arce ${ }^{a, 1}$, P.I. Diaz ${ }^{\text {b, } 1,2}$, S.P. Barros ${ }^{a, b}$, P. Galloway ${ }^{a}$, Y. Bobetsis ${ }^{a, b, 3}$, D. Threadgillc,4 \\ and S. Offenbachera,b, \\ ${ }^{a}$ Center for Oral and Systemic Diseases, NC Oral Health Institute, School of Dentistry, University \\ of North Carolina at Chapel Hill, Chapel Hill, NC, USA \\ bDepartment of Periodontology, School of Dentistry, University of North Carolina at Chapel Hill, \\ Chapel Hill, NC, USA \\ 'Department of Genetics, University of North Carolina at Chapel Hill, Chapel Hill, NC, USA
}

\begin{abstract}
Campylobacter species (C.jejuni, C. fetus) are enteric abortifacient bacteria in humans and ungulates. Campylobacter rectus is a periodontal pathogen associated with human fetal exposure and adverse pregnancy outcomes including preterm delivery. Experiments in pregnant mice have demonstrated that $C$. rectus can translocate from a distant site of infection to the placenta to induce fetal growth restriction and impair placental development. However, placental tissues from human, small-forgestational age deliveries have not been reported to harbor $C$. rectus despite evidence of maternal infection and fetal exposure by fetal IgM response. This investigation examined the temporal relationship between the placental translocation of $C$. rectus and the effects on fetal growth in mice. $\mathrm{BALB} / \mathrm{c}$ mice were infected at gestational day E7.5 to examine placental translocation of $C$. rectus by immunohistology. C. rectus significantly decreased fetoplacental weight at E14.5 and at E16.5. C. rectus was detected in $63 \%$ of placentas at E14.5, but not at E16.5. In in vitro trophoblast invasion assays, $C$. rectus was able to effectively invade human trophoblasts (BeWo) but not murine trophoblasts (SM9-1), and showed a trend for more invasiveness than C. jejuni. C. rectus challenge significantly upregulated both mRNA and protein levels of IL-6 and TNF $\alpha$ in a dose-dependent manner in human trophoblasts, but did not increase cytokine expression in murine cells, suggesting a correlation between invasion and cytokine activation. In conclusion, the trophoblast-invasive trait of $C$. rectus that appears limited to human trophoblasts may play a role in facilitating bacterial translocation and placental inflammation during early gestation.
\end{abstract}

\footnotetext{
(C) 2009 Elsevier Ireland Ltd. All rights reserved

*Corresponding author at: NC Oral Health Institute, School of Dentistry, University of North Carolina at Chapel Hill, 79 T.W. Alexander Drive, 4301 Research Commons, Research Triangle Park, NC 27709, USA. Tel.: +1 9194253596 ; fax: +1 9194253532. steve_offenbacher@dentistry.unc.edu (S. Offenbacher).

1 Authors equally contributed to this report.

${ }^{2}$ Current affiliation: Division of Periodontology, Department of Oral Health and Diagnostic Sciences, School of Dental Medicine, The University of Connecticut Health Center, Farmington, CT, USA.

${ }^{3}$ Current affiliation: Department of Periodontology, School of Dentistry, University of Athens, Athens, Greece.

${ }^{4}$ Current affiliation: Department of Microbiology, North Carolina State University, Raleigh, NC, USA.
} 


\section{Keywords}

Periodontitis; Preterm delivery; Fetal growth retardation; Campylobacter rectus; Placenta; Trophoblasts

\section{Introduction}

Campylobacter rectus is an exclusively oral Gram-negative, anaerobic and motile bacterium with a wide array of virulence factors including flagellum, surface layer proteins (S-layer), RTX-type toxins, GroELlike proteins and lipopolysaccharide (LPS) (LaGier and Threadgill, 2008; Okuda et al., 1997; Wang et al., 2000). Together with other oral anaerobic bacteria, $C$. rectus is associated with the initiation and progression of periodontal disease (Ihara et al., 2003; Socransky et al., 1998; Tanner et al., 1998). C. rectus has been implicated in the association between periodontal disease and adverse pregnancy outcomes. For example, fetal exposure to $C$. rectus has been demonstrated to be higher in preterm than in full term neonates (Madianos et al., 2001). Moreover, C. rectus count levels are higher in the oral microbiota of pregnant women with increased salivary estradiol concentrations (Yokoyama et al., 2008). Indeed, $C$. rectus seems to thrive under high concentrations of estradiol and progesterone which have been shown to significantly enhance $C$. rectus growth in vitro (Yokoyama et al., 2005). Other Campylobacter spp. including $C$. fetus and $C$. jejuni have also been reported to be associated with miscarriages, premature labor and severe perinatal infection in both humans as well as in animals (Allos, 2001; O'Sullivan et al., 1988; Simor et al., 1986; Wong et al., 1990). It is then plausible that $C$. rectus may be an important contributor to adverse pregnancy outcomes due to its ability to disseminate systemically during pregnancy.

Our laboratory has studied the effects of $C$. rectus systemic infection on the fetoplacental unit using a murine model of intra-chamber injection with live bacteria (Yeo et al., 2005). This intra-chamber model demonstrated that remote subcutaneous $C$. rectus maternal infection increases fetal resorptions and induces fetal growth restriction (Offenbacher et al., 2005). $C$. rectus infection also results in abnormal placental architecture, as evidenced by the decreased width of the vascular labyrinth and the increased width of decidual tissue in the placentas of infected growth-restricted mice (Bobetsis et al., 2007). If C. rectus disseminates systemically to reach the placenta it is then likely to interact with placental cells that express pattern recognition receptors (i.e., Toll-like receptors) (Abrahams et al., 2004), and subsequently induce a proinflammatory response that ultimately may contribute to an adverse pregnancy outcome. Indeed, recent results from our group have suggested that murine placentas from oral C. rectus-infected dams show enhanced placental TLR4 expression along with increased vasodilation in the junctional zone surrounded by focal areas of inflammatory infiltrate (Arce et al., 2009).

The in vitro interactions of $C$. rectus with placental cells are yet to be studied. Hypothetically, direct $C$. rectus contact with trophoblasts may alter gene expression and induce a proinflammatory response. C. rectus may also have the ability to invade placental trophoblasts since other Campylobacter species have been shown to readily invade host or immunocompetent cells, a feature that may play a role in their virulence potential. For example, C. jejuni invasion of enterocytes has been shown to induce oncotic changes in these cells with extensive cytoplasmic vacuolation and loss of plasma membrane integrity, an important feature in the pathogenesis of bacterial enteritis (Kalischuk et al., 2007). Moreover, bacterial invasion into mammalian cells has also been proposed as an important mechanism to evade phagocytic immune cells and allow systemic dissemination and bacterial translocation to different tissues (Li et al., 2008; Medina et al., 2003). 
In this report we evaluated the presence of $C$. rectus in the placenta of pregnant mice that were infected subcutaneously with live bacteria. We also evaluated the in vitro ability of $C$. rectus to invade human as well as murine trophoblast cells, and whether $C$. rectus infection induces changes in two important proinflammatory genes at the messenger RNA and protein levels.

\section{Methods}

\subsection{Mouse model of $C$. rectus infection}

All procedures were in accordance with the animal welfare guidelines and approved by the University of North Carolina-Chapel Hill Institutional Animal Care and Use Committee. The mouse infection model used was similar to that described before (Yeo et al., 2005). BALB/c mice were housed under controlled and standardized conditions with $12 \mathrm{~h}$ light-dark cycles. Regular mouse diet and water were provided ad libitum. Females were enrolled in the experiments at approximately 6 weeks of age and immediately had a steel chamber implanted subcutaneously. After 1 month of healing, females were mated overnight with males of the same strain. The next morning, females were removed from the male cages and examined for vaginal plugs. If a plug was found, that day was recorded as embryonic day E0.5. At E7.5, pregnant mice received an intra-chamber injection of $100 \mu \mathrm{L}$ of $10^{9} \mathrm{CFU} / \mathrm{mL}$ live $C$. rectus or saline. Mice were then sacrificed at E14.5 and fetuses $(n=15$ from 3 non-infected dams and $n=25$ from 4 infected dams) and their respective placental tissues were collected. In preliminary experiments to establish the growth restriction model we collected weight data for fetoplacental units obtained from 27 non-infected dams and 32 infected dams sacrificed at E16.5. For histological analysis, placentas were collected and bisected sagitally then fixed in $4 \%$ paraformaldehyde and embedded in paraffin. Sections $(6 \mu \mathrm{m})$ were stained using standard hematoxylin and eosin protocols and imaged using a Nikon Microphot-FXA Microscope equipped with a QImaging Micropublisher CCD camera. Morphometric measurements of the area occupied by each placental layer, namely decidua, spongiotrophoblast layer and labyrinth, were conducted using the "Image J" software (http://rsb.info.nih.gov/ij/). For detection of $C$. rectus in placental tissues, placentas from 2 control mice $(n=10)$ and 2 infected mice $(n=11)$ from gestational day E14.5 were examined by immunostaining. Briefly, sections were deparaffinized, re-hydrated in ethanol/ $\mathrm{H}_{2} \mathrm{O}$ washes and permeabilized by incubation in $0.2 \%$ Triton $\mathrm{X}$ in PBS. Slides were then incubated for $1 \mathrm{~h}$ in blocking buffer (5\% BSA, 1\% goat serum and $0.2 \%$ Triton- $\mathrm{X}$ in PBS) and then incubated overnight at $4{ }^{\circ} \mathrm{C}$ with an FITCconjugated anti-Campylobacter antibody (Kirkegaard \& Perry Labs, MD) and Texas Redconjugated Phalloidin (Invitrogen, CA). Slides were washed several times in PBS and visualized with a Zeiss LSM5 Pascal Confocal Laser Scanning Microscope and 63× na 1.4 plan apo and 100× na 1.3 plan neofluar lenses. Images were captured using the LSM510 software. Images obtained from preliminary E16.5 experiments were included for comparison purposes.

\subsection{Mammalian cell lines}

The human trophoblast cell line BeWo (ATCC CCL-98) derived from a human choriocarcinoma (Pattillo and Gey, 1968) and the mouse trophoblast cell line SM9-1 (a kind gift from JS Hunt, University of Kansas Medical Center, Kansas City, KS) derived from a gestational day 9 Swiss-Webster mouse placenta (Bowen and Hunt, 1999) were used for invasion experiments and cytokine assays. The Madin-Darby canine kidney epithelial cell line MDCK (ATCC CCL-34) was used as a positive control for $C$. jejuni invasion as documented elsewhere (Wine et al., 2008). BeWo cells were grown in Ham's F12K medium with $2 \mathrm{mM}_{\mathrm{L}-}$ glutamine adjusted to contain 10\% fetal bovine serum (FBS) according to ATCC propagation instructions. SM9-1 cells were grown in RPMI-1640 supplemented with $1 \mathrm{mM}$ sodium pyruvate, $5 \times 10^{-5} \mathrm{M} 2$-mercaptoethanol and penicillin/streptomycin. Culture medium for MDCK cells was Eagle's Minimum Essential Medium with 10\% FBS. All cells were grown 
in T-25 flasks (Corning Inc. Life Sciences, Lowell, MA) or onto cover slips placed at the bottom of a well in 24 well plates for invasion analysis. All cells were grown at $37{ }^{\circ} \mathrm{C}$ in $5 \% \mathrm{CO}_{2}$.

\subsection{Bacterial cultures}

Campylobacter rectus strain 314 or Campylobacter jejuni strain ATCC 43457 were used for infection experiments. C. rectus 314 is an oral isolate from a periodontal disease patient. $C$. rectus was grown under anaerobic conditions at $37^{\circ} \mathrm{C}$ on Trypticase soy broth agar (Becton Dickinson and Company, Cockeysville, MD) supplemented with $0.2 \%$ yeast extract, $0.3 \%$ Phytone peptone, $0.2 \% \mathrm{NaCl}, 0.3 \%$ ammonium formate, $0.4 \%$ sodium fumarate and $0.4 \% \mathrm{~L}-$ asparagine, adjusted to $\mathrm{pH}$ 7.8. C. jejuni was grown on Mueller Hinton agar (Oxoid, Cambridge, UK) plates at $37^{\circ} \mathrm{C}$ under a microaerophilic atmosphere.

\subsection{Invasion assays}

Mammalian cells were grown onto cover slips in 24 well plates until 80-90\% confluency. Bacterial strains were harvested from agar plates after $72 \mathrm{~h}$ incubation ( $48 \mathrm{~h}$ incubation yielded adequate growth for $C$. jejuni) and resuspended in tissue culture medium without antibiotics to an optical density of $1.00(600 \mathrm{~nm})$. Mammalian cell monolayers were also washed 3 times with cell culture medium without antibiotics prior to inoculation with bacteria. Bacterial cells were added to obtain a multiplicity of infection (MOIs) of 100 bacteria/mammalian cell, after which plates were centrifuged at $250 \times g$ for $5 \mathrm{~min}$, incubated for $5 \mathrm{~h}$ at $37^{\circ} \mathrm{C}$ in $10 \% \mathrm{CO}_{2}$ and washed with PBS. This time point and MOI were chosen for quantification as pilot experiments showed that a similar number of $C$. rectus cells were found intracellularly at 5, 12 and $24 \mathrm{~h}$ (data not shown). For visualization of adhesion/invasion, infected and non-infected controls were then washed 3 times with PBS to remove non-adhered bacteria and fixed with $4 \%$ paraformaldehyde for $30 \mathrm{~min}$ at room temperature. After washing twice with PBS, cells were permeabilized with $0.2 \%$ Triton X-100 in PBS for 15 min. Blocking buffer (5\% BSA, $1 \%$ goat serum and $0.2 \%$ Triton-X-100) was added for $1 \mathrm{~h}$ and cells were incubated overnight at $4{ }^{\circ} \mathrm{C}$ with an FITC-conjugated anti-Campylobacter antibody and Texas Red-conjugated Phalloidin. Cells were then washed several times with PBS. Cover slips were mounted on glass slides and observed using a Zeiss LSM5 Pascal Confocal Laser Scanning Microscope and 63×na 1.4 planapo and 100×na 1.3 plan neofluar lenses. Random fields were captured using the LSM510 software. Quantification of bacterial adhesion and invasion was done manually using the LSM510 software and the ortho view to navigate the entire stack of images visualizing $x, y$ and $z$ planes. Image stacks of up to 8 random fields were used for quantification of invasion/ adhesion by confocal microscopy.

\subsection{Proinflammatory gene expression and cytokine assays}

Infected cells at MOI 50,100 and 500 during $12 \mathrm{~h}$ and non-infected controls were washed 3 times with PBS to remove non-adhered cells. The MOIs were determined by performing preliminary experiments testing over a range of MOI that extended from 5 to $1000 \mathrm{MOI}$. Additional experiments were also performed to include ultrapure $E$. coli LPS (0111:B4 strain, Invivogen, San Diego, CA) using $1 \mu \mathrm{g} /$ well as positive control for proinflammatory gene expression. Total RNA was isolated from cells with the use of the miRNeasy Mini Kit (Qiagen, Valencia, CA). $1 \mu \mathrm{g}$ of total RNA was reversely transcribed using the Omniscript system (Qiagen). Real-time PCR was performed with $1 \mu \mathrm{L}$ of the cDNA reaction in a 7500 Sequence Detection System (ABI Prism, Applied Biosystems, Foster City, CA). TaqMan pre-inventoried assays for human (Hs00985639_m1) and mouse (Mm00446190_m1) Interleukin 6 (IL-6) as well as for human (Hs99999043_m1) and mouse (Mm99999068_m1) tumor necrosis factor alpha (TNF $\alpha$ ) were used for relative gene expression quantification. Reactions were performed in duplicates and two independent assays were carried out for each gene. The $18 \mathrm{~S}$ ribosomal RNA subunit gene and the glyceraldehyde-3-phosphate dehydrogenase (gapdh) gene were 
used as endogenous housekeeping controls for human (BeWo) and murine (SM9-1) trophoblast cells, respectively. Results were evaluated using the delta-delta $\mathrm{Ct}$ method, where delta $\mathrm{Ct}$ was calculated as (gene of interest $\mathrm{Ct}$ ) - (housekeeping gene $\mathrm{Ct}$ ), and the relative quantity of mRNA expression was calculated by the delta-delta $\mathrm{Ct}$ as $2-[$ (infected sample delta $\mathrm{Ct})-($ non-infected sample delta $\mathrm{Ct}$ )]. The quantification of IL-6 and TNF $\alpha$ in cell supernatants was performed by means of xMAP multiplexing cytokine assays. Briefly, cell supernatants were collected after timed infection, centrifuged at $1500 \times \mathrm{g}$ for $5 \mathrm{~min}$ and then frozen until analysis. Multianalyte kits for human and mouse IL- 6 and TNF $\alpha$ were used following the manufacturer's instructions (Fluorokine MAP Kits, R\&D systems, Minneapolis, MN).

\subsection{Statistical analysis}

A minimal sample size of 8 mice per group was calculated [power $(1-\beta)$ of $>0.90 \%$ with alphaerror threshold of $(\alpha)=0.05$ ] based on our previous results on fetal growth restriction after $C$. rectus systemic infection at E16.5 (Yeo et al., 2005). Continuous variables were expressed as means and standard deviations. Mean placental weight values for control and infected mice were compared using the unpaired Student's $t$-test. The detection frequency of C. rectus in infected and non-infected murine placentas was compared by using the Fisher's exact test. Counts of $C$. rectus invasion/adhesion in cells were compared using the unpaired Student's $t$ test. mRNA fold differences and protein concentration differences were compared using oneway ANOVA. The threshold for statistical significance was set at a $P$-value less than 0.05 . All analyses were performed using SAS v.9.2 (SAS Institute, Cary, NC).

\section{Results}

C. rectus induces growth restriction and translocates from a distant site of infection to the murine placenta in vivo. C. rectus infection impaired the development of the fetoplacental unit at day E14.5 and E16.5 (Table 1). At E14.5, mice infected with C. rectus had a significantly lighter placental weight $(0.104 \pm 0.02 \mathrm{~g})$ and fetoplacental weight $(0.236 \pm 0.04 \mathrm{~g}, n=25)$ compared to the average placental weight $(0.118 \pm 0.02 \mathrm{~g}, P=0.03)$ and fetoplacental weight $(0.262 \pm 0.03, n=15, P=0.02)$ of non-infected mice. At E16.5, fetoplacental weight showed a similar trend to that of E14.5 $(0.570 \pm 0.004 \mathrm{~g}$ in $C$. rectus-infected mice vs. $0.612 \pm 0.004 \mathrm{~g}$ in non-infected mice) and this difference was even more significant $(P>0.0001)$; however, we observed a different trend for placental weight at E16.5, where $C$. rectus-infected placentas were heavier than non-infected controls $[0.14 \pm 0.005 \mathrm{~g}$ vs. $0.132 \pm 0.002 \mathrm{~g}$, respectively $(P>$ $0.0001)$ ], probably because infected placentas presented higher cellular inflammatory infiltration leading to placental edema (Offenbacher et al., 2005). Also, in contrast to our previous findings at E16.5 (Bobetsis et al., 2007), the morphometric analysis of the placentas from infected mice showed no significant differences in the mean percentage area of each of the placental zones when compared to controls at E14.5 [49 \pm 8.45 vs. $53.8 \pm 14.0$, respectively $(P=0.08)]$; however, a trend for an increase in the size of the decidua layer in infected placentas from fetuses with the lowest weight was noted. We also observed focal areas of inflammatory infiltrate in the junctional zone of infected placentas as reported previously for E16.5 (data not shown).

Immunofluorescent labeling of $C$. rectus on murine placental tissues and trophoblasts in culture is depicted in Fig. 1. C. rectus was detected in 7 out of 11 stained placentas (63.6\%) from infected mice (Fig. 1b and c) and in none of the 10 placentas from non-infected controls (Fig. 1a) $(P=0.004)$ at E14.5. The presence of bacterial cells was not observed in the majority of the scanned fields in infected placental tissues, as the bacteria were rather scarcely distributed and seen as single or coupled bacteria cells. No bacterial cells were observed in murine placentas analyzed at E16.5 (data not shown). 
C. rectus adheres and invades human trophoblasts. C. rectus adhesion and invasion, as visualized by confocal microscopy, was evaluated in murine and human trophoblasts. In addition, we compared the invasiveness of the oral $C$. rectus to a highly invasive intestinal pathogenic strain of $C$. jejuni. Epithelial cells (MDCK) served as positive control for Campylobacter invasion. Extracellular and intercellular localization of $C$. rectus without cytoplasmic invasion was evident in murine trophoblasts (Fig. 1e). At the same microbial load, there was much greater accumulation of $C$. jejuni extracellularly (Fig. 1f) without appreciable evidence of intracellular invasion for either $C$. jejuni or $C$. rectus. However, both bacteria were able to penetrate cell junctions and $C$. jejuni was more efficient than $C$. rectus at doing so (Table 2). In contrast, both $C$. rectus and $C$. jejuni were able to invade and localize intracellularly in the human trophoblast BeWo cells, as well as in the positive control MDCK cells (Fig. 1g-1). In all cases, $C$. jejuni adhered, penetrated through cell junctions and invaded with significantly more efficiency than $C$. rectus, except for intracellular invasion of the human trophoblast cell line (BeWo) in which $C$. rectus showed a non-statistically significant trend for greater invasion over $C$. jejuni $(P=0.08)$.

C. rectus upregulates IL- 6 and TNF $\alpha$ gene expression in human trophoblasts. Table 3 illustrates the mRNA relative gene fold differences of $C$. rectus-infected cells vs. non-infected controls. Human BeWo cell responses to the positive control (E. coli LPS) were upregulated for TNF $\alpha$ (67.63 \pm 0.65$)$ and IL-6 (11.47 \pm 0.28$)$; however, gene expression responses to LPS were particularly low for the murine SM9-1 cells for both IL-6 (0.74 \pm 0.28$)$ and TNF $\alpha(1.12 \pm 0.34)$. A statistically significant trend was also observed in $C$. rectus-infected cells, in which dosedependent responses of proinflammatory genes were observed for IL-6 $(P<0.05)$ and TNF $\alpha$ $(P<0.001)$ in human BeWo cells, but conversely relative gene differences in the murine SM9-1 cells were low for IL-6 and TNF $\alpha$ (Table 3).

In vitro C. rectus infection upregulates proinflammatory cytokine expression in human trophoblasts. Fig. 2 depicts the cytokine concentration differences in cell supernatants from infection experiments at $12 \mathrm{~h}$. Both human IL-6 and TNF $\alpha$ were increased in a MOI-dependent manner. Protein concentration differences were significantly different for human IL-6 ( $P<$ $0.0001)$ and TNF $\alpha(P<0.0001)$. Murine proinflammatory cytokines were not detected in cell supernatants after infection with $C$. rectus.

\section{Discussion}

The chamber infection murine model used in this report aims to simulate bacterial chronic exposure in the murine host mimicking the repeated systemic challenge that would be associated with a chronic oral infection in humans. Here we report fetoplacental unit growth restriction at gestational day E14.5 (Table 1) along with evidence of $C$. rectus translocation to and invasion of the murine placenta in vivo. The human clinical isolate $C$. rectus 314 was detected in $64 \%$ of murine placentas after 7 days post-infection at E14.5 by immunohistology (Fig. 1a-c), but C. rectus was not detected at E16.5 using the same technique (data not shown). Interestingly, this finding could mean that intact bacteria can be found in the placenta at E14.5 but only bacterial DNA is detected at E16.5 by means of nested PCR amplifications (Bobetsis et al., 2007). Since immunofluorescence not only detects viable bacteria but also dead bacterial cells, such differences may be partially explained by $C$. rectus losing viability due to limited growth conditions or due to immune clearance. Interestingly, $C$. rectus detection in placental tissues from human, small-for-gestational age deliveries has not been reported yet, despite evidence of a remarkable degree of fetal antibody seropositivity (cord blood IgM) to $C$. rectus (Madianos et al., 2001).

Host cell invasion is an important feature in the pathogenesis of many bacteria, including members of the Campylobacteraceae family. Campylobacter invasion into host cells has been 
studied mainly with the enteric pathogen $C$. jejuni (Fields and Thompson, 2008; KrauseGruszczynska et al., 2007). The invasiveness of $C$. jejuni in cultured epithelial cell lines has been proposed to correlate with colonization efficiency and disease outcome (Bacon et al., 2000; Hanel et al., 2004). However, to the best of our knowledge this investigation represents the first study to present evidence of in vitro $C$. rectus invasion in trophoblastic cells and this invasion process appears to be specific for human trophoblasts (Fig. 1). The invasiveness of C. rectus evaluated in this study via confocal microscopy appears to be within the range reported for other Campylobacter species in terms of quantity (0.01-2 bacteria per cell) (Biswas et al., 2004). In these studies the number of internalized bacteria seems to increase with greater concentrations reaching a plateau at a MOI of 100-200; however, even with the most invasive strains only one to three bacteria are internalized per cell (Friis et al., 2005).

Even though $C$. jejuni showed significantly greater activity in terms of cellular adhesion and paracellular translocation, this study supports the finding that scarce bacterial numbers of $C$. rectus or $C$. jejuni get internalized in human BeWo trophoblasts using similar MOIs. However, we found that $C$. rectus preferentially invades human trophoblasts in vitro (Table 2), which may indicate that $C$. rectus specifically targets the human placental trophoblast and thus contributes to the apparent fetoplacental tropism proposed in humans (Madianos et al., 2001). Furthermore, the invasion efficiency of $C$. rectus appeared to be greater than the invasiveness of an established placental pathogen such as $C$. jejuni, at least for the $C$. jejuni strain tested. Similar routes of infection from mucosal surfaces to other tissues have been described for different $C$. jejuni strains, which have been shown to internalize into host cells localizing intracellularly (van Alphen et al., 2008) and also to translocate through cell junctions disrupting tight junction protein components (Chen et al., 2006). Either bacterial paracellular (through junctions between cells) translocation or transcellular (via the cell cytoplasm) internalization could potentially be useful for $C$. rectus to cross the oral epithelial barrier and to migrate to distant sites including the developing fetoplacental unit. The ability to invade human trophoblasts and localize in their cytoplasm may also confer $C$. rectus protection from immune surveillance while in the immunologically protected placental tissue, as demonstrated with other abortifacient bacteria such as Listeria monocytogenes (Bakardjiev et al., 2006).

This study also demonstrated that in response to $C$. rectus challenge a proinflammatory response is induced in human trophoblastic cells. A clear dose-dependent (MOI) proinflammatory activity was observed in the human BeWo cell line (Table 3 and Fig. 2) as evidenced by the gene expression and protein upregulation of IL- 6 and TNF $\alpha$, two important members of the cluster of proinflammatory cytokines involved in pathogenesis of chorioamnionitis, preterm labor, low birth weight and preterm premature rupture of membranes associated with intraamniotic infections (Gomez et al., 1997). Furthermore, among different placental cell populations, trophoblasts in particular have been proposed to play a key role in coordinating placental immune responses during implantation and pregnancy maintenance (Mor, 2008). Trophoblasts are also thought to be primary sentinel cells that are involved in microbial clearance preventing microbial translocation of infectious agents from mother to fetus (Levy, 2007).

The results obtained with human trophoblasts contrast to those obtained with murine trophoblasts, which were unresponsive to the $C$. rectus in vitro challenge and did not show significant proinflammatory activity (Table 3). Even though the SM9-1 cell line has been reported to express TNF $\alpha$ mRNA (Sharma, 1998), these murine trophoblasts derived from Swiss mice did not offer a significant proinflammatory response when challenged by $C$. rectus. This finding is also supported by the observation that SM9-1 murine trophoblasts were not responsive to $E$. coli LPS challenge $(1 \mu \mathrm{g} / \mathrm{mL})$, although cells remained viable based upon proliferation assays (data not shown). Moreover, C. rectus did not appear invasive in the mouse trophoblast cultures, but did co-localize at regions of intercellular junctions suggesting that 
this human pathogen may use a paracellular pathway to effect translocation to promote murine growth restriction. In fact, we have observed the bacteria confined to blood vessels in the labyrinth rather than in the placental tissues, suggesting a different invasion mechanism in vivo. Similarly, it is known that differences exist in the response of human and murine epithelial cells to other Campylobacter species, as evidenced by differences in the expression of genes involved in growth, transcription and steroid biosynthesis in human colonic epithelia vs. unresponsive murine colonic cells (Rinella et al., 2006). Others have also reported that $C$. jejuni, which normally does not bind to Chinese hamster ovary cells, binds avidly when these cells are transfected with the human alpha1,2-fucosyltransferase gene, thus further demonstrating the specificity of Campylobacter spp. for human cells (Ruiz-Palacios et al., 2003). To summarize, the differences between human and murine responses suggest that $C$. rectus may have a recognition system that is specific for human trophoblasts which could potentially enhance tropism and immune activation in the human host.

In conclusion, our studies demonstrated that $C$. rectus has the ability to translocate in vivo from a distant site of infection to the fetoplacental unit. The ability of $C$. rectus to invade human trophoblast cell lines and localize intracellularly as well as its ability to increase the expression of important proinflammatory cytokines highlights its potential to be an important contributor to adverse pregnancy outcomes associated with periodontal disease in humans.

\section{Acknowledgments}

We would like to thank Dr. Robert Bagnell and Victoria J. Madden for imaging assistance, and Dr. David Barrow for laboratory support. This work was supported by the National Institutes of Health grants RO1-DE-12453, P-60DE-13079, and U01 DE14577.

\section{References}

Abrahams VM, Bole-Aldo P, Kim YM, Straszewski-Chavez SL, Chaiworapongsa T, Romero R, Mor G. Divergent trophoblast responses to bacterial products mediated by TLRs. J. Immunol 2004;173(7): 4286-4296. [PubMed: 15383557]

Allos BM. Campylobacter jejuni infections: update on emerging issues and trends. Clin. Infect. Dis 2001;32(8):1201-1206. [PubMed: 11283810]

Arce RM, Barros SP, Wacker B, Peters B, Moss K, Offenbacher S. Increased TLR4 expression in murine placentas after oral infection with periodontal pathogens. Placenta 2009;30(2):156-162. [PubMed: 19101032]

Bacon DJ, Alm RA, Burr DH, Hu L, Kopecko DJ, Ewing CP, Trust TJ, Guerry P. Involvement of a plasmid in virulence of Campylobacter jejuni 81-176. Infect. Immun 2000;68(8):4384-4390. [PubMed: 10899834]

Bakardjiev AI, Theriot JA, Portnoy DA. Listeria monocytogenes traffics from maternal organs to the placenta and back. PLoS Pathog 2006;2(6):e66. [PubMed: 16846254]

Biswas D, Niwa H, Itoh K. Infection with Campylobacter jejuni induces tyrosine-phosphorylated proteins into INT-407 cells. Micro-biol. Immunol 2004;48(4):221-228.

Bobetsis YA, Barros SP, Lin DM, Weidman JR, Dolinoy DC, Jirtle RL, Boggess KA, Beck JD, Offenbacher S. Bacterial infection promotes DNA hypermethylation. J. Dent. Res 2007;86(2):169174. [PubMed: 17251518]

Bowen JA, Hunt JS. Expression of cell adhesion molecules in murine placentas and a placental cell line. Biol. Reprod 1999;60(2):428-434. [PubMed: 9916011]

Chen ML, Ge Z, Fox JG, Schauer DB. Disruption of tight junctions and induction of proinflammatory cytokine responses in colonic epithelial cells by Campylobacter jejuni. Infect. Immun 2006;74(12): 6581-6589. [PubMed: 17015453]

Fields JA, Thompson SA. Campylobacter jejuni CsrA mediates oxidative stress responses, biofilm formation, and host cell invasion. J. Bacteriol 2008;190(9):3411-3416. [PubMed: 18310331] 
Friis LM, Pin C, Pearson BM, Wells JM. In vitro cell culture methods for investigating Campylobacter invasion mechanisms. J. Microbiol. Methods 2005;61(2):145-160. [PubMed: 15722140]

Gomez R, Romero R, Edwin SS, David C. Pathogenesis of preterm labor and preterm premature rupture of membranes associated with intraamniotic infection. Infect. Dis. Clin. North Am 1997;11(1):135176. [PubMed: 9067790]

Hanel I, Muller J, Muller W, Schulze F. Correlation between invasion of Caco-2 eukaryotic cells and colonization ability in the chick gut in Campylobacter jejuni. Vet. Microbiol 2004;101(2):75-82. [PubMed: 15172689]

Ihara H, Miura T, Kato T, Ishihara K, Nakagawa T, Yamada S, Okuda K. Detection of Campylobacter rectus in periodontitis sites by monoclonal antibodies. J. Periodont. Res 2003;38(1):64-72. [PubMed: 12558939]

Kalischuk LD, Inglis GD, Buret AG. Strain-dependent induction of epithelial cell oncosis by Campylobacter jejuni is correlated with invasion ability and is independent of cytolethal distending toxin. Microbiology 2007;153(Pt 9):2952-2963. [PubMed: 17768238]

Krause-Gruszczynska M, Rohde M, Hartig R, Genth H, Schmidt G, Keo T, Konig W, Miller WG, Konkel ME, Backert S. Role of the small Rho GTPases Rac1 and Cdc42 in host cell invasion of Campylobacter jejuni. Cell Microbiol 2007;9(10):2431-2444. [PubMed: 17521326]

LaGier MJ, Threadgill DS. Identification of novel genes in the oral pathogen Campylobacter rectus. Oral Microbiol. Immunol 2008;23(5):406-412. [PubMed: 18793364]

Levy O. Innate immunity of the newborn: basic mechanisms and clinical correlates. Nat. Rev. Immunol 2007;7(5):379-390. [PubMed: 17457344]

Li L, Michel R, Cohen J, Decarlo A, Kozarov E. Intracellular survival and vascular cell-to-cell transmission of Porphyromonas gingivalis. BMC Microbiol 2008;8:26. [PubMed: 18254977]

Madianos PN, Lieff S, Murtha AP, Boggess KA, Auten RL Jr. Beck JD, Offenbacher S. Maternal periodontitis and prematurity. Part II. Maternal infection and fetal exposure. Ann. Periodontol 2001;6 (1):175-182. [PubMed: 11887461]

Medina E, Goldmann O, Toppel AW, Chhatwal GS. Survival of Streptococcus pyogenes within host phagocytic cells: a pathogenic mechanism for persistence and systemic invasion. J. Infect. Dis 2003;187(4):597-603. [PubMed: 12599076]

Mor G. Inflammation and pregnancy: the role of toll-like receptors in trophoblast-immune interaction. Ann. N. Y. Acad. Sci 2008;1127:121-128. [PubMed: 18443339]

O'Sullivan AM, Dore CJ, Coid CR. Campylobacters and impaired fetal development in mice. J. Med. Microbiol 1988;25(1):7-12. [PubMed: 2961890]

Offenbacher S, Riche EL, Barros SP, Bobetsis YA, Lin D, Beck JD. Effects of maternal Campylobacter rectus infection on murine placenta, fetal and neonatal survival, and brain development. J. Periodontol 2005;76(11 Suppl.):2133-2143. [PubMed: 16277586]

Okuda K, Kigure T, Yamada S, Kaneko T, Ishihara K, Miura T, Kato T, Takazoe I. Role for the S-layer of Campylobacter rectus ATCC33238 in complement mediated killing and phagocytic killing by leukocytes from guinea pig and human peripheral blood. Oral Dis 1997;3(2):113-120. [PubMed: 9467352]

Pattillo RA, Gey GO. The establishment of a cell line of human hormone-synthesizing trophoblastic cells in vitro. Cancer Res 1968;28(7):1231-1236. [PubMed: 4299001]

Rinella ES, Eversley CD, Carroll IM, Andrus JM, Threadgill DW, Threadgill DS. Human epithelialspecific response to pathogenic Campylobacter jejuni. FEMS Microbiol. Lett 2006;262(2):236-243. [PubMed: 16923081]

Ruiz-Palacios GM, Cervantes LE, Ramos P, Chavez-Munguia B, Newburg DS. Campylobacter jejuni binds intestinal $\mathrm{H}(\mathrm{O})$ antigen (Fuc alpha 1, $2 \mathrm{Gal}$ beta 1, 4GlcNAc), and fucosyloligosaccharides of human milk inhibit its binding and infection. J. Biol. Chem 2003;278(16):14112-14120. [PubMed: 12562767]

Sharma RK. Mouse trophoblastic cell lines. I. Relationship between invasive potential and TGF-beta 1. In Vivo 1998;12(5):431-440. [PubMed: 9827348]

Simor AE, Karmali MA, Jadavji T, Roscoe M. Abortion and perinatal sepsis associated with campylobacter infection. Rev. Infect. Dis 1986;8(3):397-402. [PubMed: 3523697] 
Socransky SS, Haffajee AD, Cugini MA, Smith C, Kent RL Jr. Microbial complexes in subgingival plaque. J. Clin. Periodontol 1998;25(2):134-144. [PubMed: 9495612]

Tanner A, Maiden MF, Macuch PJ, Murray LL, Kent RL Jr. Microbiota of health, gingivitis, and initial periodontitis. J. Clin. Periodontol 1998;25(2):85-98. [PubMed: 9495607]

van Alphen LB, Bleumink-Pluym NM, Rochat KD, van Balkom BW, Wosten MM, van Putten JP. Active migration into the subcellular space precedes Campylobacter jejuni invasion of epithelial cells. Cell Microbiol 2008;10(1):53-66. [PubMed: 18052944]

Wang B, Kraig E, Kolodrubetz D. Use of defined mutants to assess the role of the Campylobacter rectus S-layer in bacterium-epithelial cell interactions. Infect. Immun 2000;68(3):1465-1473. [PubMed: 10678961]

Wine E, Chan VL, Sherman PM. Campylobacter jejuni mediated disruption of polarized epithelial monolayers is cell-type specific, time dependent, and correlates with bacterial invasion. Pediatr. Res 2008;64(6):599-604. [PubMed: 18679160]

Wong SN, Tam AY, Yuen KY. Campylobacter infection in the neonate: case report and review of the literature. Pediatr. Infect. Dis. J 1990;9(9):665-669. [PubMed: 2235192]

Yeo A, Smith MA, Lin D, Riche EL, Moore A, Elter J, Offenbacher S. Campylobacter rectus mediates growth restriction in pregnant mice. J. Periodontol 2005;76(4):551-557. [PubMed: 15857095]

Yokoyama M, Hinode D, Masuda K, Yoshioka M, Grenier D. Effect of female sex hormones on Campylobacter rectus and human gingival fibroblasts. Oral Microbiol. Immunol 2005;20(4):239243. [PubMed: 15943769]

Yokoyama M, Hinode D, Yoshioka M, Fukui M, Tanabe S, Grenier D, Ito HO. Relationship between Campylobacter rectus and periodontal status during pregnancy. Oral Microbiol. Immunol 2008;23 (1):55-59. [PubMed: 18173799] 

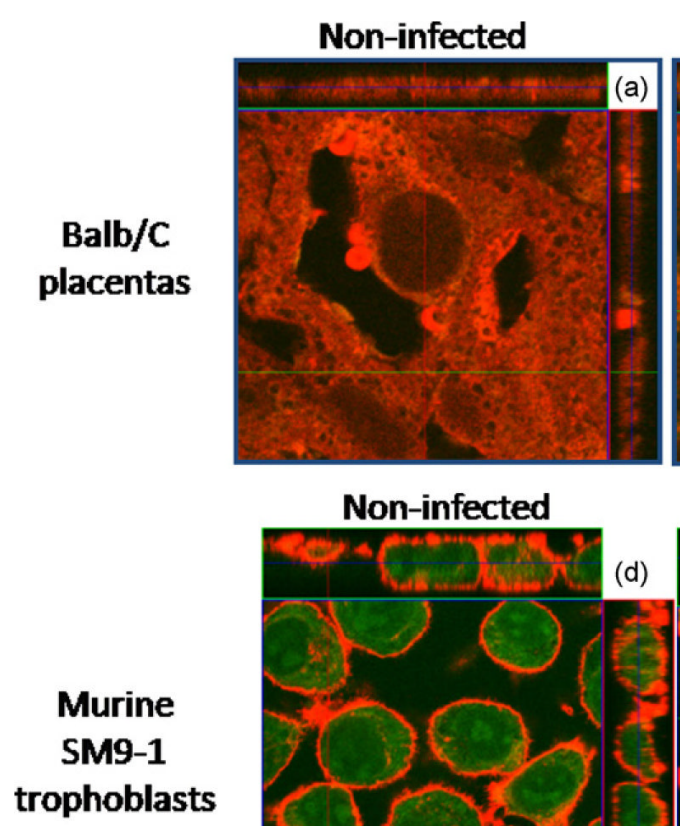
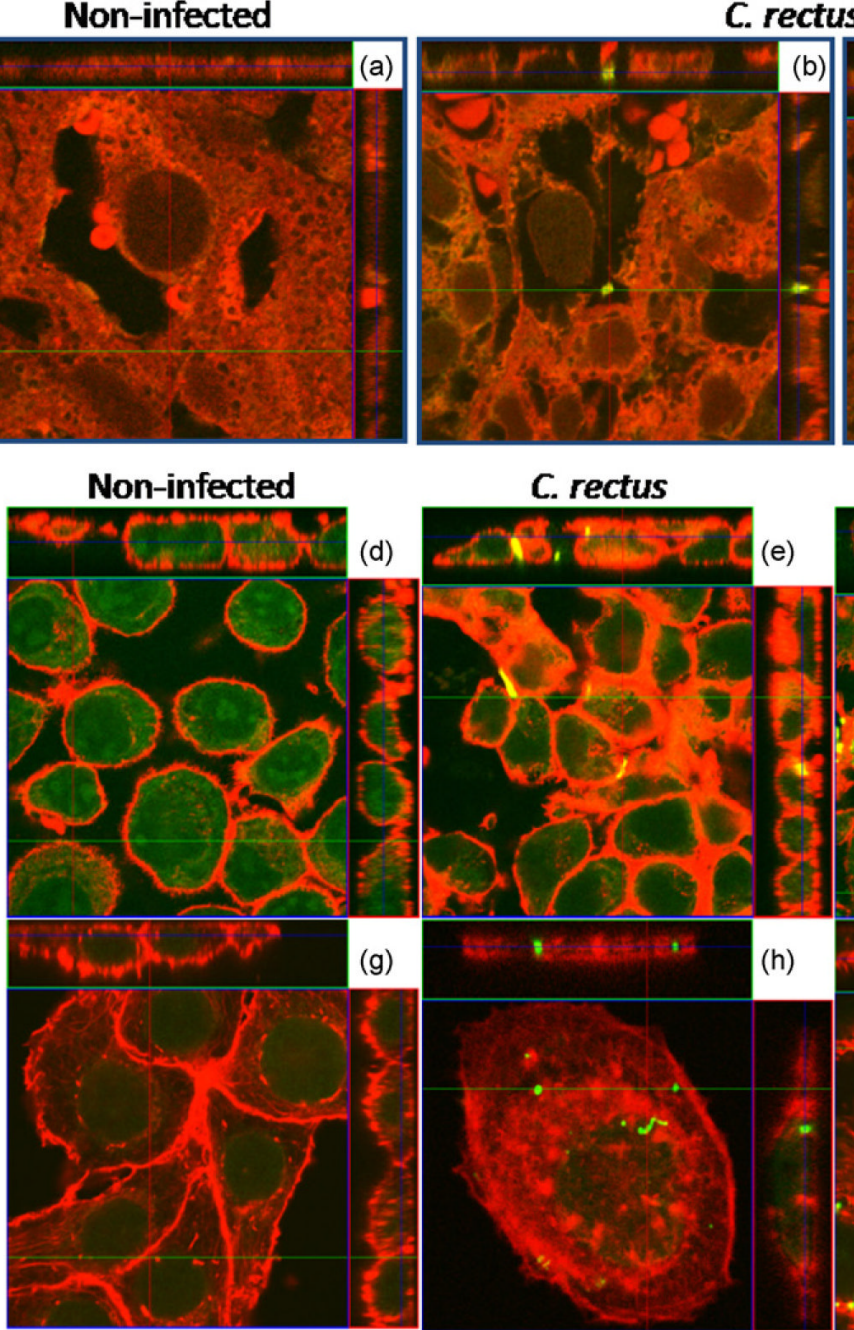

C. rectus-infected
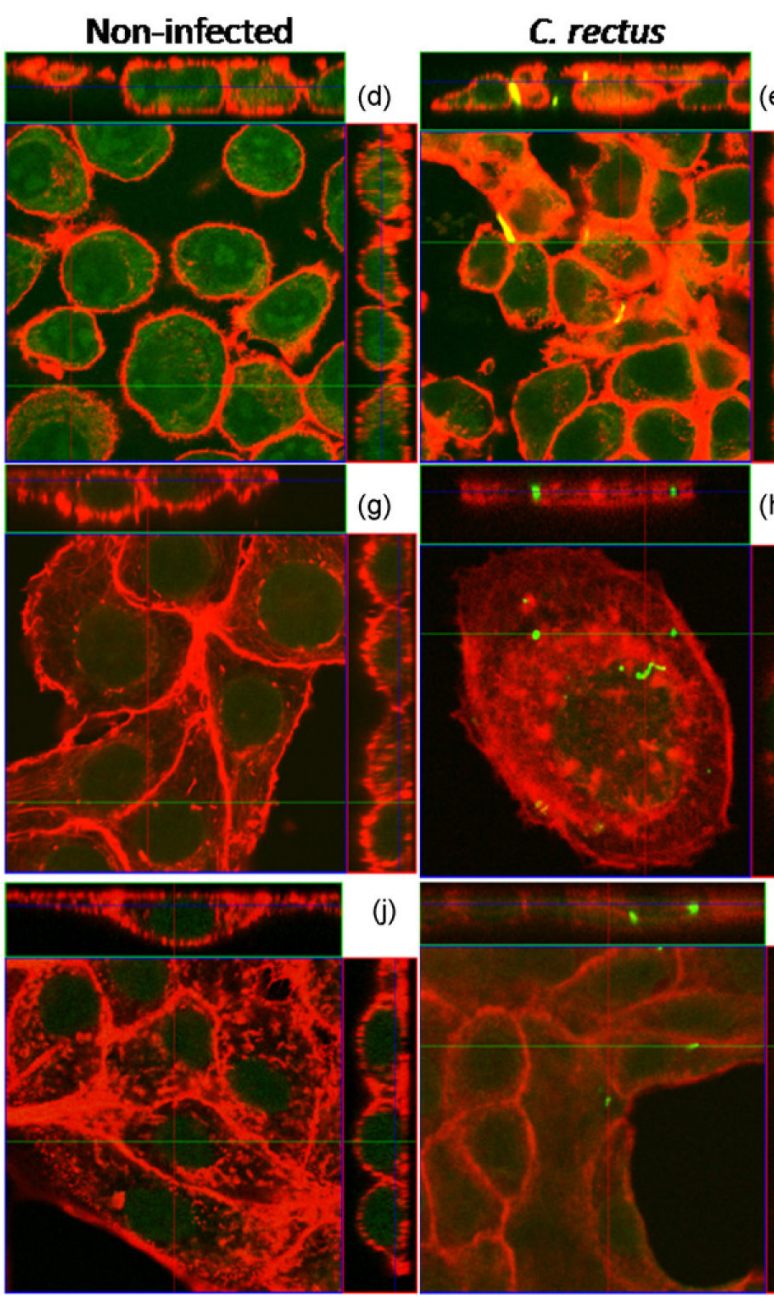

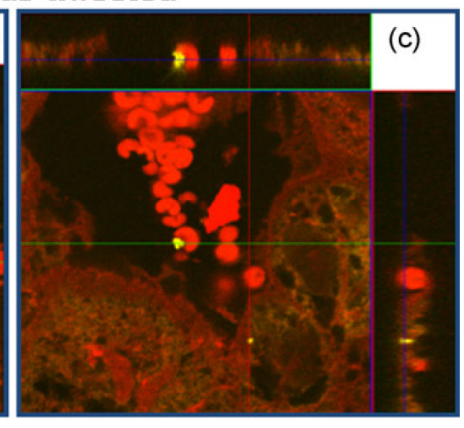

\section{C. jejuni}

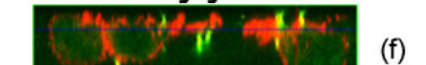

(e)

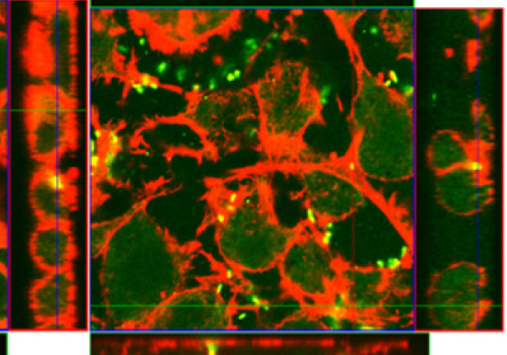

(h)

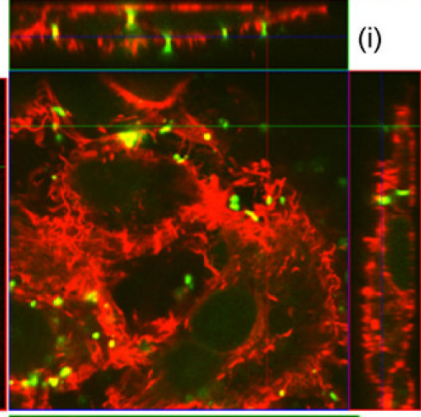

(k)

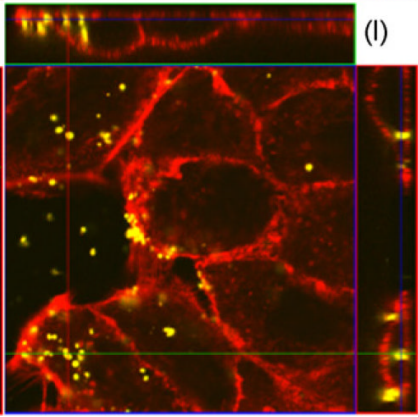

Fig. 1.

C. rectus invasiveness in murine placentas and mammalian trophoblasts. Immunofluorescence analysis of murine placentas: $(\mathrm{a}-\mathrm{c})$ representative images in murine placentas from noninfected (a) and infected mice (b and c). C. rectus was seen in placentas of infected mice (64\%) but not in the placentas of control mice. The presence of bacterial cells was rather scarce and was not observed in the majority of the scanned fields: $(\mathrm{d}-\mathrm{i})$ representative images from in vitro trophoblastic cell infection experiments and non-infected controls. Bacteria were found extracellularly attached to murine trophoblasts, or present in the cell junctions within cells (e) without apparent evidence of cytoplasmic invasion (f). Conversely, C. rectus and C. jejuni were detected intracellularly in the human trophoblast BeWo cells (h and i). (j-l) The canine 
epithelial MDCK cells served as positive control target cells using a highly invasive intestinal pathogenic strain of $C$. jejuni. For all image stacks, red stain corresponds to F-actin stained with Texas Red-conjugated Phalloidin. Green-yellow fluorescent cells represent bacteria stained with an FITC-conjugated Campylobacter-specific antibody. Magnifications: [a-c] at $63 \times$ na 1.4 plan apo and [d-1] at $100 \times$ na 1.3 plan neo fluar lenses. (For interpretation of the references to color in this figure legend, the reader is referred to the web version of the article.) 
Human IL-6

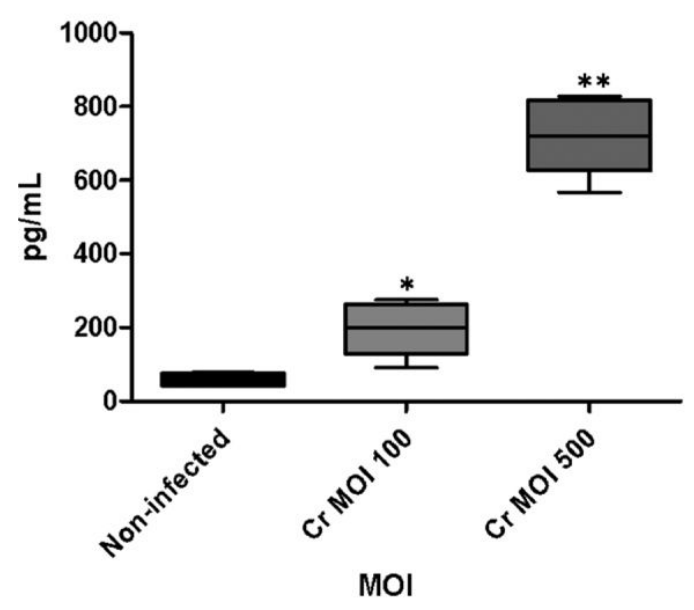

Human TNF $\alpha$

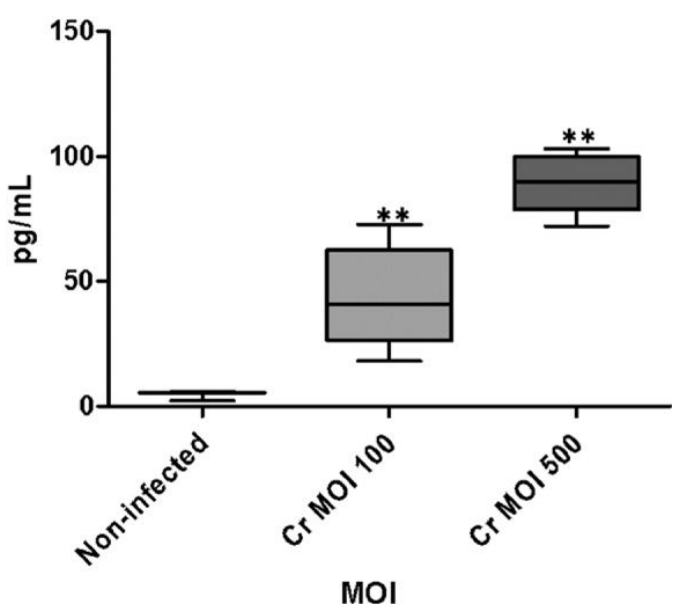

Fig. 2.

Proinflammatory cytokine expression in human trophoblasts after in vitro C. rectus infection. Box plots depict mean \pm SD cytokine concentration values. Cell supernatants were harvested and cytokines were quantified by $\times$ MAP multiplexing. Human IL- 6 and TNF $\alpha$ were significantly increased in a MOI-dependent manner. $* P<0.05$ and $* * P<0.0001$. 


\section{Table 1}

Growth restriction in fetoplacental units at day E14.5 and E16.5 after C. rectus infection. Data represent mean values $\pm \mathrm{SD}$ per group. Pregnant mice received an intra-chamber injection of live $C$. rectus or saline at E7.5. Mice were sacrificed at E14.5 and 16.5 for the analysis of their fetoplacental tissues. Mean placental and fetoplacental weight were significantly different between non-infected controls and $C$. rectus-infected mice. $n$ values correspond to the number of fetuses or placentas analyzed.

\begin{tabular}{llcc}
\hline Gestational day/weight & Non-infected control & C. rectus-infected & $P$-Value \\
\hline E14.5 & & & \\
$\quad$ Placental weight & $0.118 \pm 0.020(n=15)$ & $0.104 \pm 0.020(n=25)$ & 0.03 \\
Fetoplacental weight & $0.262 \pm 0.030(n=15)$ & $0.236 \pm 0.040(n=25)$ & 0.02 \\
E16.5 & & & \\
Placental weight & $0.132 \pm 0.002(n=143)$ & $0.14 \pm 0.005(n=105)$ & $<0.0001$ \\
Fetoplacental weight & $0.612 \pm 0.004(n=143)$ & $0.570 \pm 0.004(n=105)$ & $<0.0001$ \\
\hline
\end{tabular}




\section{Table 2}

Quantification of adhesion and intercellular/intracellular localization of $C$. rectus and $C$. jejuni in trophoblast cells in vitro. Data represent the mean number of bacterial cells counted in the entire stack of images in up to 8 random fields per sample (Fig. 1). C. jejuni and C rectus adhered to SM9-1 murine trophoblast cells but invasion was negligible. In contrast, both $C$. jejuni and $C$. rectus invaded the human trophoblast cell line BeWo and the canine epithelial cell MDCK $(P<0.05)$. It was noted that in most cases $C$. jejuni adhesion/invasion of cells was significantly greater than that of $C$. rectus. However, $C$. rectus showed a non-significant trend of higher invasiveness counts in BeWo cells when compared to $C$. jejuni $(P=0.08)$.

\begin{tabular}{|l|l|c|c|c|}
\hline \multirow{2}{*}{ Cell line } & Bacteria & Bacteria adhered/cell & $\begin{array}{c}\text { Bacteria colocalizing with actin (cell } \\
\text { junctions) per cell }\end{array}$ & Invasive bacteria/cell (cytoplasmic) \\
\hline \multirow{2}{*}{ SM9-1 } & C. rectus 314 & $0.50 \pm 0.41^{*}$ & $0.44 \pm 0.10^{*}$ & $0.00 \pm 0.00$ \\
\cline { 2 - 5 } & C. jejuni 43457 & $4.79 \pm 2.82^{*}$ & $1.53 \pm 0.71^{*}$ & $0.00 \pm 0.00$ \\
\hline \multirow{2}{*}{ BEWO } & C. rectus 314 & $0.43 \pm 0.52^{*}$ & $0.08 \pm 0.11^{* *}$ & $5.51 \pm 3.18$ \\
\cline { 2 - 5 } & C. jejuni 43457 & $5.30 \pm 1.75^{*}$ & $2.83 \pm 0.32^{* *}$ & $2.96 \pm 0.77$ \\
\hline \multirow{2}{*}{ MDCK } & C. rectus 314 & $0.26 \pm 0.36^{*}$ & $0.10 \pm 0.12^{*}$ & $0.69 \pm 0.25^{*}$ \\
\cline { 2 - 5 } & C. jejuni 43457 & $3.83 \pm 2.40^{*}$ & $1.40 \pm 0.65^{*}$ & $1.84 \pm 1.19^{*}$ \\
\hline
\end{tabular}

${ }^{*} P<0.05$

** $P<0.01$ 


\section{Table 3}

Relative proinflammatory gene expression in trophoblastic cultures after $C$. rectus infection in vitro. Data represent mean fold changes \pm SD normalized to non-infected controls. Cells were infected at MOI 50, 100 and 500 during $12 \mathrm{~h}$ and the relative expression of human or murine IL- 6 and TNF $\alpha$ was quantified by qPCR.

\begin{tabular}{crr}
\hline Gene & MOI & mRNA expression fold differences \\
\hline Human $I L 6$ & 50 & $2.75 \pm 0.38$ \\
& 100 & $4.18 \pm 0.85^{*}$ \\
& 500 & $6.39 \pm 0.61^{* *}$ \\
& 50 & $10.9 \pm 0.54^{* *}$ \\
Human $T N F \alpha$ & 100 & $24.6 \pm 0.55^{* *}$ \\
& 500 & $56.4 \pm 1.96^{* *}$ \\
& 50 & $0.5 \pm 6.88$ \\
Murine $I L 6$ & 100 & $4.15 \pm 1.87$ \\
& 500 & $2.31 \pm 2.10$ \\
& 50 & $1.23 \pm 0.64$ \\
Murine $T N F \alpha$ & 100 & $1.0 \pm 0.34$ \\
& 500 & $1.24 \pm 0.49$ \\
\hline * & \\
&
\end{tabular}

